\title{
The loop-forming method as a useful technique to rotate the endoscopic insertion tube shaft
}

Authors

Institutions
Tomoaki Suga', Takuma Okamura', Norikazu Arakura², Eiji Tanaka

${ }^{1}$ Department of Gastroenterology, Shinshu University School of Medicine, Matsumoto, Japan

${ }^{2}$ Endoscopic Examination Center, Shinshu University Hospital, Matsumoto, Japan submitted 2. January 2015 accepted after revision 27. October 2015

Bibliography Dol http://dx.doi.org/ 10.1055/s-0041-109082 Published online: 11.1.2016 Endoscopy International Open 2016; 04: E170-E174

(c) Georg Thieme Verlag KG Stuttgart · New York E-ISSN 2196-9736

\section{Corresponding author} Tomoaki Suga, MD Department of

Gastroenterology

Shinshu University School of Medicine

3-1-1 Asahi

Matsumoto 390-8621

Japan

Fax: +81-263-32-9412

sugatomo@shinshu-u.ac.jp
Background and study aims: Gastroenterological endoscopists are instructed to bring the target to the 6-o'clock position when they take a biopsy specimen, use a snare, or cut a target organ. This action is performed primarily by rotating the shaft of the endoscopic insertion tube, which can be difficult in some situations when existing endoscopic methods are used. We previously described a method for optimal rotation of the endoscopic insertion tube shaft, called the loopforming method (LFM). The present study aimed to validate this procedure and confirm the usefulness of the LFM for leftward rotation of the shaft. Participants and methods: The LFM was specifi-

\section{Introduction}

$\nabla$

Gastroenterological endoscopists are instructed to adjust the instrument channel outlet to the target when they take a biopsy specimen, puncture, use a snare, or cut a target organ [1,2]. Doing so allows a clear line of sight between the object lens and the target, thereby making treatment easier and safer $(\bullet$ Fig. 1 a, $\bullet$ Fig. 1 b). Such a maneuver is performed primarily by rotating the shaft of the endoscopic insertion tube, which can be difficult in some situations when existing endoscopic methods are used. We earlier described and analyzed a method for optimal rotation of the endoscopic insertion tube shaft, called the loop-forming method (LFM) [3]. The present study aimed to validate the usefulness of this technique. cally taught to 28 gastroenterological endoscopists, and the angle of rotation was measured before and after they received instruction in this method.

Results: The LFM significantly increased the average angle of leftward rotation from $266^{\circ}$ to $327^{\circ}$. Moreover, whereas the instrument channel inlet tended to move away from the right hand of an operator using a conventional endoscopy method, it remained closely accessible to the right hand of an examiner using the LFM.

Conclusions: The LFM has the potential to make endoscopic procedures easier and safer.

\section{Participants and methods}

$\nabla$

Endoscopic insertion tube shaft rotation

Conventional method to rotate the endo-

scope insertion tube shaft leftward

$(\bullet$ Fig.2a, $\diamond$ Fig.2b)

Many endoscopists rotate the shaft of the insertion tube to the left by moving the control section downward and leftward. As the shaft rotates to the left, the control section tends to project farther away from the right hand. This may make it difficult to operate instruments through the instrument channel in some cases.

\section{Mechanics of the loop-forming method}

\section{$(\odot$ Fig.3a, $\odot$ Fig.3b, $\odot$ Fig.3c)}

As the insertion tube is twisted to the left, it gradually forms a loop. After a rotation angle of $360^{\circ}$, it will have formed a perfect loop and be difficult to twist further. In the LFM, the examiner rotates the shaft of the endoscopic insertion tube by using the left hand to form a loop. 


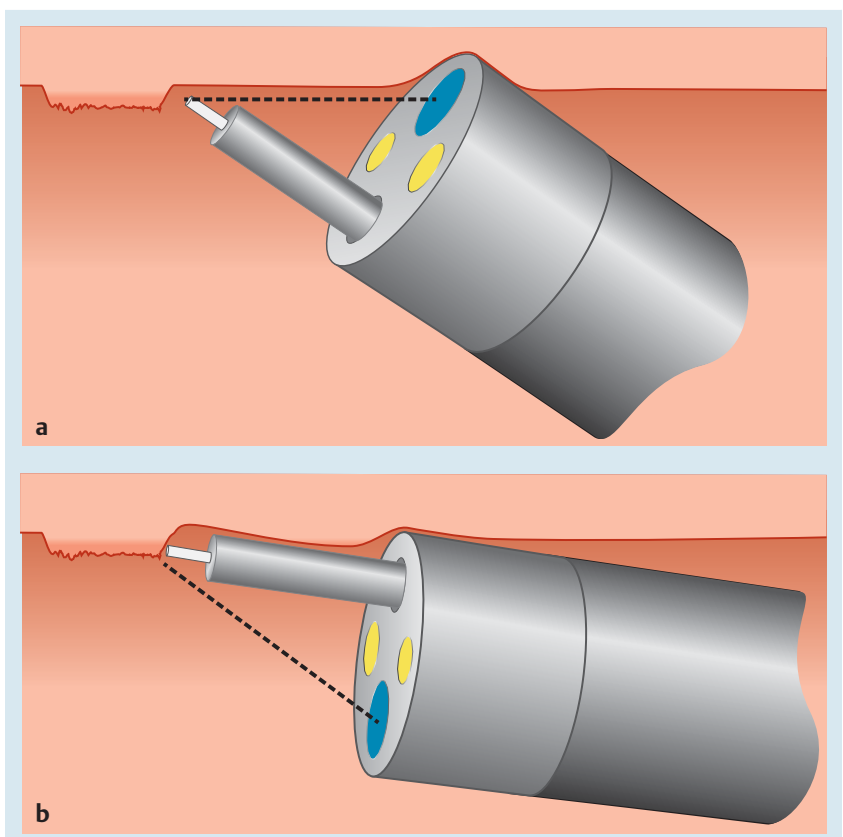

Fig. 1 a It may be difficult to treat the target on the upper wall because of hampered visibility. $\mathbf{b}$ Bringing the target to the 6 -o'clock position creates a clear line of sight between the object lens and the target.

\section{Clinical application of the loop-forming method}

\section{$(\bullet$ Fig. 4 a, $\diamond$ Fig. 4b, $\bullet$ Fig.4c)}

The examiner stands facing the patient, who is typically lying on the left side, and moves the left hand forward and downward while holding the control section. The right hand lightly supports the insertion tube without grasping it. This technique cannot be readily employed with an endoscope that has a long shaft, such as a colonoscope.

\section{Verification of the usefulness of the loop-forming method \\ Device for measuring the shaft rotation angle}

We constructed a device to measure the rotation angle of the endoscopic insertion tube shaft. A cylinder with a protractor attached at one end was fixed to the bottom of a larger cylinder $50 \mathrm{~cm}$ in length. The endoscope was inserted into the bottom of the cylinder and set at an origin point ( $\boldsymbol{\bullet}$ Fig.5). We considered this point to be the position where the vertical line on a monitor and the actual vertical line (extending from 12 to 6 o'clock) would be in alignment. We measured the rotation angle on a monitor screen ( $\bullet$ Fig. 6, $\odot$ Video 1 ). A gastroscope (GIF-Q240; Olympus, Tokyo, Japan) was used in this study.
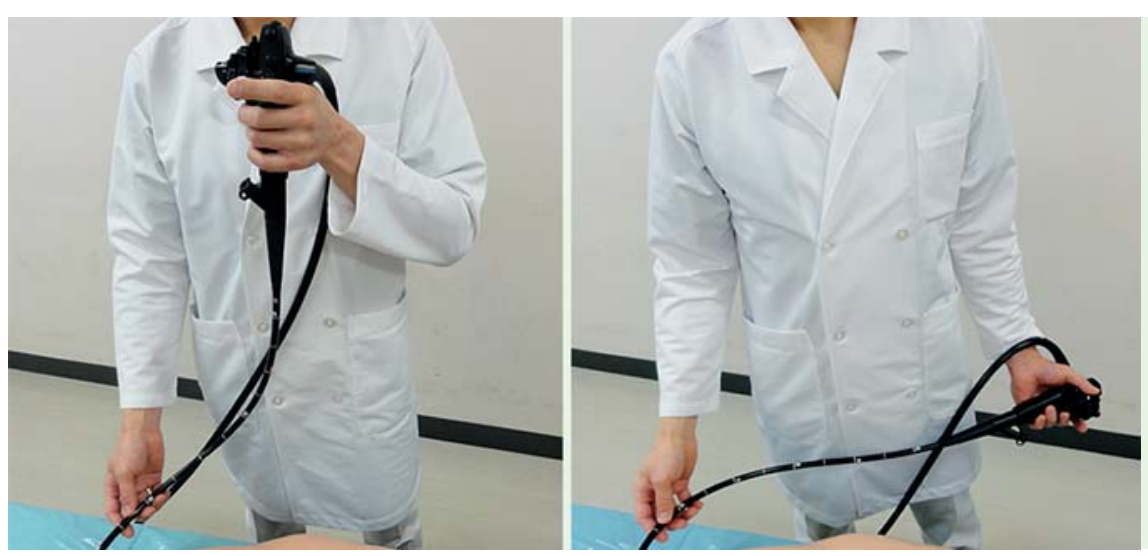

Fig. 2 Conventional method of rotating the endoscopic insertion tube shaft to the left. a Starting position without rotation. $\mathbf{b}$ Maximum shaft rotation position. The inlet of the instrument channel is facing the floor.

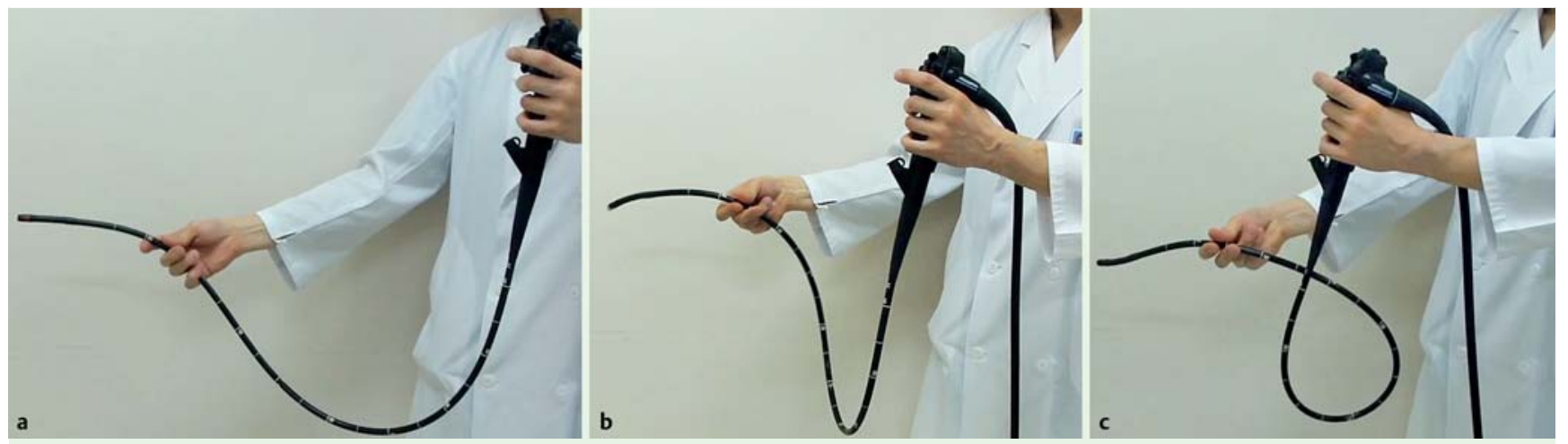

Fig. 3 Mechanics of the loop-forming method. a Starting position without rotation. $\mathbf{b}$ As the insertion tube is twisted to the left, it gradually forms a loop. c When it is twisted $360^{\circ}$, the insertion tube will have formed a perfect loop. 


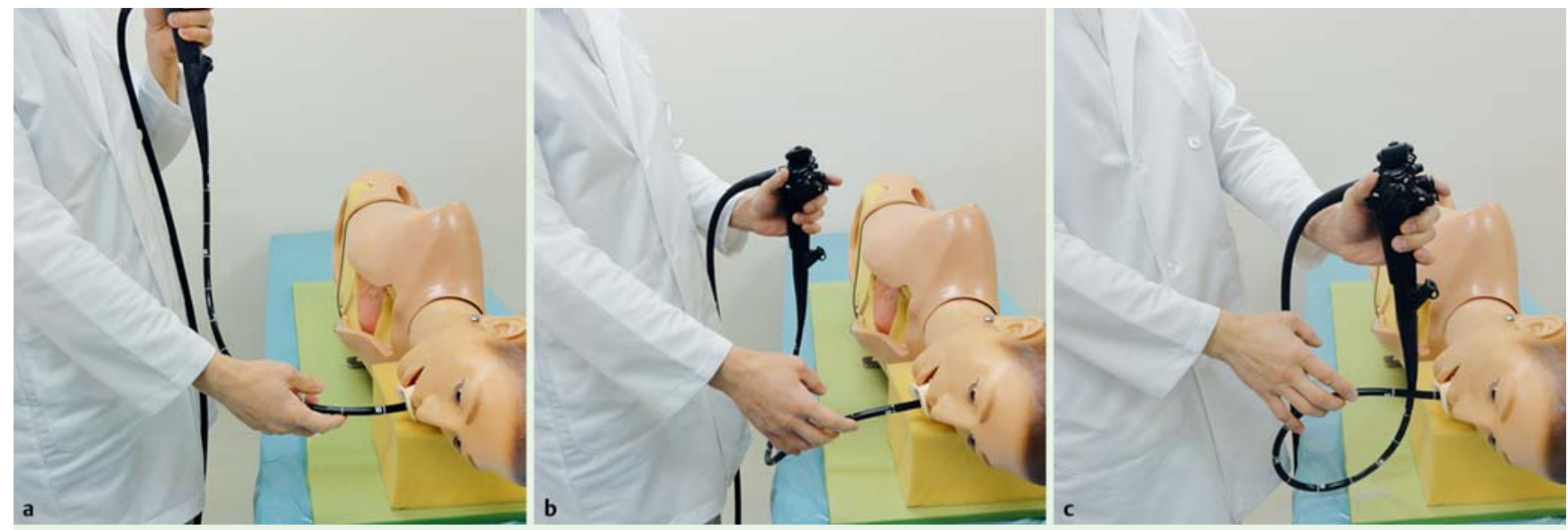

Fig.4 Clinical application of the loop-forming method. a Starting position without rotation. $\mathbf{b}$ To rotate the shaft of the endoscopic insertion tube, the examiner moves the left hand forward and downward while grasping the control section. The right hand lightly supports the insertion tube without grasping it. c To obtain maximum rotation, the examiner shortens the distance between the right and left hands.

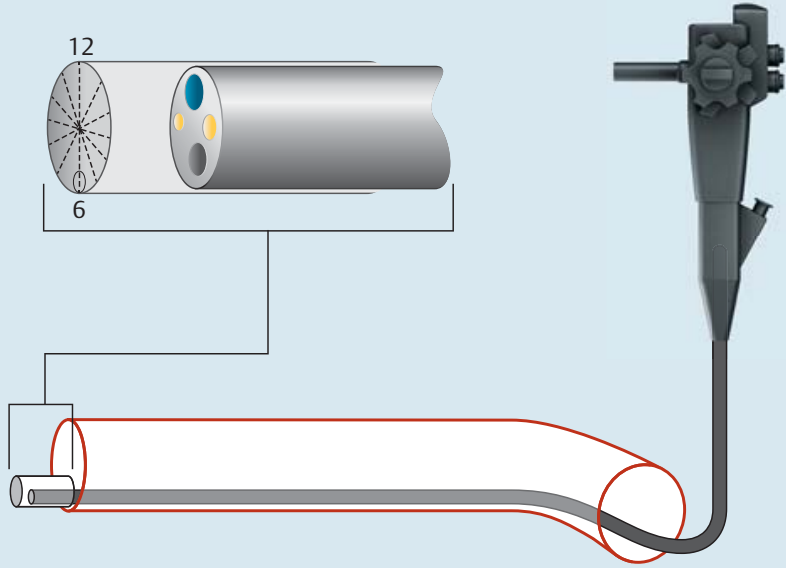

Fig. 5 Device for measuring the rotation angle of the endoscopic insertion tube shaft with an endoscope placed inside.

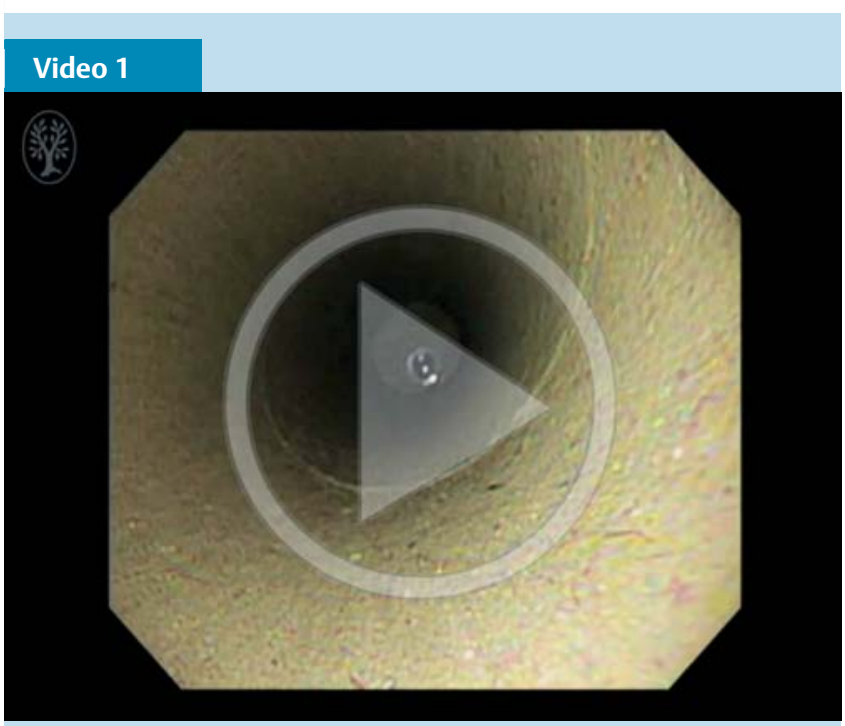

An examiner is inserting the endoscope into the measuring device. At the bottom of the fixed smaller cylinder, the examiner can be seen to rotate the shaft to the left for a total of $90^{\circ}, 180^{\circ}$, and $270^{\circ}$ as measured by a protractor attached to the bottom of the device. Online content including video sequences viewable at: http://dx.doi.org/10.1055/s-0041-109082

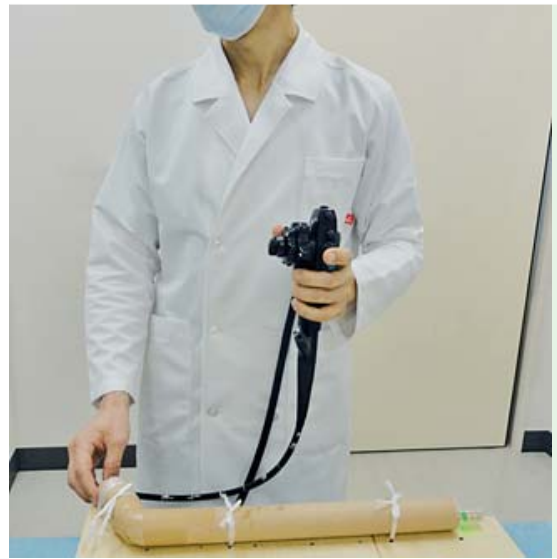

Fig. 6 The measuring device is first fixed on a bed. The examiner and a judge measure rotation on a monitor. This position is $180^{\circ}$ of leftward rotation with the loop-forming method.

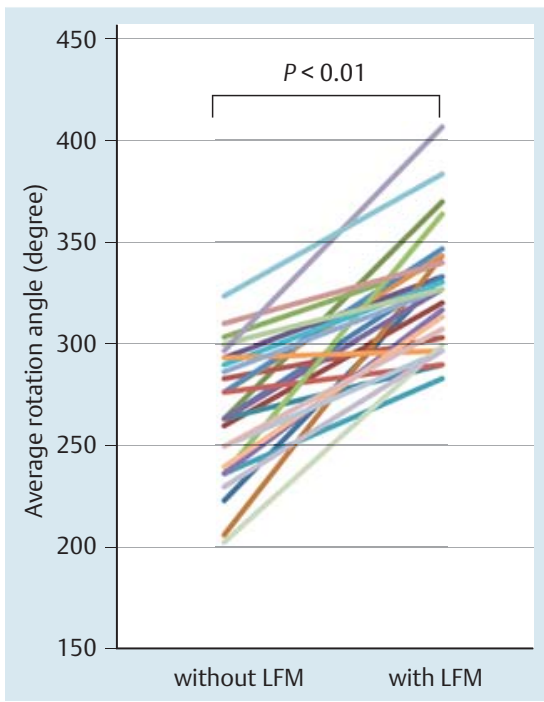

Fig. 7 Average rotation angles of the endoscopic insertion tube shaft to the left before and after a lecture on the loop-forming method (LFM). By using the LFM, all endoscopists increased their average leftward rotation angle, and the increase was significant $(P<0.01)$. 


\section{Participants and methods}

The study enrolled 28 endoscopists working in our endoscopic examination center $(-$ Table $\mathbf{1})$. At first, each participant rotated the shaft of the endoscopic insertion tube to the left in a conventional fashion, and the angle from the point of origin to the point of maximum rotation was evaluated with the measuring device three times. The endoscopists were strictly ordered not to twist the insertion tube with the right hand. Afterward, they attended a lecture on the LFM and then repeated the same measurement experiment while using the new technique. They were again instructed not to twist the insertion tube with the right hand and not to tilt the control section of the endoscope. The control unit of the endoscope was held on each participant's right side. Statistical analysis was performed with Wilcoxon's rank sum test.

Table 1 Characteristics of 28 participants enrolled in a study of the loopforming method as a useful technique to rotate the endoscopic insertion tube shaft.

\begin{tabular}{|lc|}
\hline Men/women, $\mathrm{n}(\%)$ & $24(86) / 4(14)$ \\
\hline Age, median (range), y & $31(26-45)$ \\
\hline Experience as an endoscopist, median (range), y & $4(0.5-19)$ \\
\hline
\end{tabular}

\section{Results}

\section{$\nabla$}

All of the endoscopists lowered the control section of endoscope to the left when they initially rotated the shaft of the endoscopic insertion tube leftward ( $\bullet$ Fig. 2). We noted that the LFM enabled all participants to improve their average rotation angle to the left; the average leftward rotation angle increased significantly from $266^{\circ}$ (range $203^{\circ}-323^{\circ}$ ) with the conventional method to $327^{\circ}$ (range $\left.283-407^{\circ}\right)$ with the LFM $(P<0.01)$ ( $\odot$ Fig. 7).

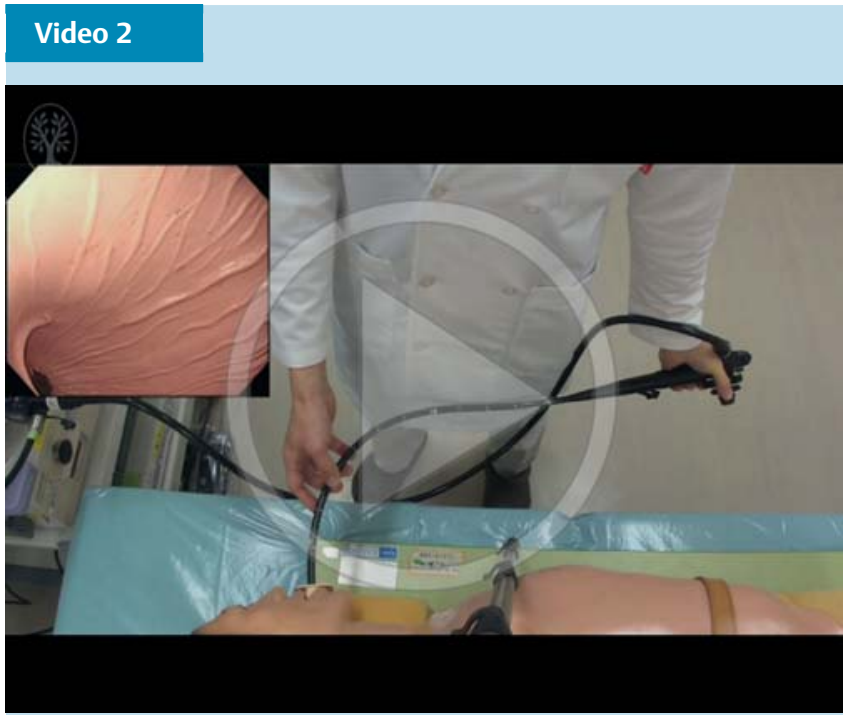

Comparison of methods to rotate the endoscopic insertion tube shaft to the left. The maximum rotation angle obtained with the conventional method can be achieved with only moderate movement when the loop-forming method is used. Online content including video sequences viewable at: http://dx.doi.org/10.1055/s-0041-109082

\section{Discussion}

$\nabla$

Operation of a gastroscope can be broadly divided into three components: pushing the insertion tube in and pulling it out, controlling angulation, and rotating the shaft of the insertion tube. Rotation is an important endoscopic movement that allows a clear line of sight between the object lens and the target, thereby making procedures easier and safer. Although doctors routinely perform examinations with a gastroscope worldwide, there has been little discussion on the ideal way to operate the device. Many operators naturally bend the endoscopic insertion tube to some extent to rotate the shaft. However, no studies have precisely analyzed the mechanism of shaft rotation, and so a bending method to rotate the shaft of the endoscopic insertion tube has not been formally introduced until now. We earlier devised a simple model of the LFM to establish an optimal bending method for shaft rotation [3]. The device for measuring the shaft rotation angle has enabled us to analyze and discuss this technique in greater detail.

All of the participants in this study initially tilted the control section of the endoscope downward and leftward when rotating the endoscopic insertion tube shaft to the left. The average leftward rotation angle was $266^{\circ}$ with the conventional method, which increased by approximately $60^{\circ}$ with the LFM. The shape of the loop and degree of wrist flexion for each endoscopist likely accounted for the variance in rotation angles. The LFM is most applicable when the insertion tube length into the patient's body is between 0 and approximately $55 \mathrm{~cm}$ as the endoscope used in the present study does not allow the insertion tube shaft to be bent to a diameter of less than $12 \mathrm{~cm}$. Under these conditions, similar results can be expected in live patients undergoing gastric endoscopic procedures in a wide range of body positions because the effectiveness of the LFM depends on the configuration of the loop outside the body.

Although the conventional endoscope rotation style may be a more natural way to rotate the shaft, we have shown that it has a clear disadvantage for leftward rotation compared with the LFM. Furthermore, the instrument channel inlet became dis-

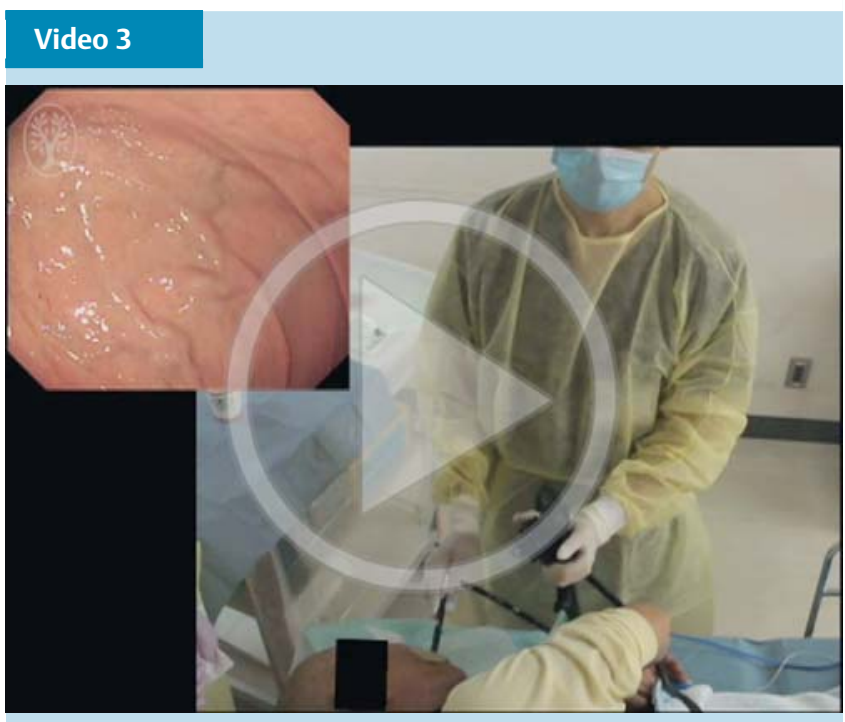

The endoscopist can examine all areas of the fornix and cardia while in a steady position when using the loop-forming method. Online content including video sequences viewable at: http://dx.doi.org/10.1055/s-0041109082 
tanced from the right hand when the shaft was rotated fully to the left with the ordinary method ( $\nabla$ Fig. $\mathbf{2 b}$ ). Thus, although a conventional approach may be adequate for screening endoscopy, handling instruments through the instrument channel may become problematic with the examiner in such a strained posture. This is a primary reason why the leftward rotation angle is reduced when endoscopists use instruments with the conventional method. On the other hand, the leftward rotation angle was significantly increased with the LFM, and most of the operative angles $\left(0-270^{\circ}\right)$ remained available with the examiner in a comfortable posture without right-hand support of the insertion tube shaft.

Approximately $360^{\circ}$ of total rotation (right plus left) angle are needed for some endoscopic treatments. Full rotation is especially useful during endoscopic injection sclerotherapy for esophageal varices [3]. Although near-maximum leftward rotation ( $\bullet$ Fig. 2 b) may be required to obtain such an angle with a conventional method, only moderate leftward rotation ( $\bullet$ Fig. $\mathbf{4 b}$ ) is needed to obtain this angle with the LFM ( $\bullet$ Video 2 ). The LFM is also very useful during endoscopic screening procedures, especially in the fornix and cardia, by allowing operators to examine all areas while in a steady position ( Video 3 ).

In conclusion, we recommend specific instruction in the LFM technique for all endoscopists to increase the ease and safety of biopsy and therapeutic procedures.

\section{Competing interests: None}

\section{References}

1 Peter VD. Colonoscopic polypectomy and associated techniques. World J Gastroenterol 2010; 16: 3630-3637

2 Uraoka T, Ramberan H, Matsuda T et al. Cold polypectomy techniques for diminutive polyps in the colorectum. Dig Endosc 2014; 26: 98-103

3 Suga T, Nagaya T, Arakura N. Loop-forming method: new method for endoscopic insertion tube shaft rotation. Dig Endosc 2014; 26: $122-$ 123 